\title{
Restricted isotype, distinct variable gene usage, and high rate of gp120 specificity of HIV-1 envelope-specific B cells in colostrum compared with those in blood of HIV-1-infected, lactating African women
}

CR Sacha $^{1}$, N Vandergrift ${ }^{1}$, TL Jeffries Jr ${ }^{1}$, E McGuire $^{1}$, GG Fouda ${ }^{1}$, B Liebl ${ }^{1}$, DJ Marshall ${ }^{1}$, TC Gurley ${ }^{1}$, L Stiegel $^{1}$, JF Whitesides ${ }^{1}$, J Friedman ${ }^{1}$, A Badiabo ${ }^{1}$, A Foulger ${ }^{1}$, NL Yates ${ }^{1}$, GD Tomaras ${ }^{1}$, TB Kepler ${ }^{2}$, HX Liao ${ }^{1}$, BF Haynes ${ }^{1}$, MA Moody ${ }^{1,3}$ and SR Permar ${ }^{1,3}$

A successful HIV-1 vaccine must elicit immune responses that impede mucosal virus transmission, though functional roles of protective HIV-1 Envelope (Env)-specific mucosal antibodies remain unclear. Colostrum is a rich source of readily accessible mucosal $B$ cells that may help define the mucosal antibody response contributing to prevention of postnatal HIV-1 transmission. To examine the HIV-1 Env-specific colostrum B-cell repertoire, single B cells were isolated from 17 chronically HIV-infected, lactating women, producing 51 blood and 39 colostrum HIV-1 Env-specific B-cell antibodies. All HIV-1 Env-specific colostrum-derived antibodies were immunoglobulin (Ig)G1 isotype and had mean heavy chain complementarity-determining region 3 (CDR3) lengths and mutation frequencies similar to those isolated from blood. However, variable heavy chain $\left(\mathrm{V}_{\mathrm{H}}\right)$ gene subfamily $1 \sim 69$ usage was higher among colostrum than blood HIV-1 Env-reactive antibodies ( $49 \%$ vs. $20 \%, P=0.006$, Fisher's exact test). Additionally, more HIV-1 Env-specific colostrum antibodies were gp120 specific than those isolated from blood (44\% vs. $16 \%, P=0.005$, Fisher's exact test). One cross-compartment HIV-1 Env-specific clonal B-cell lineage was identified. These unique characteristics of colostrum B-cell antibodies suggest selective homing of HIV-1-specific IgG1-secreting memory B cells to the mammary gland and have implications for targeting mucosal B-cell populations by vaccination.

\section{INTRODUCTION}

The HIV-1 epidemic continues to be fueled by 2.3 million new infections annually, ${ }^{1}$ highlighting the critical need for an effective HIV-1 vaccine. As HIV-1 transmission occurs mainly at mucosal surfaces, effective anti-HIV-1 antibody responses at the portal of viral entry may reduce the risk of virus acquisition. Yet, it remains unclear whether effective HIV-1 vaccination necessitates protective mucosal antibody responses. Furthermore, whether mucosal antibody responses were potentially responsible for the moderate protection observed in the RV144 vaccine trial remains unknown. ${ }^{2}$ Determining the role of HIV-1 Env-specific mucosal immunoglobulin (Ig)G and IgA antibodies in preventing mucosal HIV-1 transmission is therefore an essential step toward HIV-1 vaccine design and evaluation.

With recent advances in recombinant antibody technology, numerous HIV-1-neutralizing monoclonal antibodies (mAbs) have been produced from B lymphocytes isolated from peripheral blood of HIV-1-infected individuals. ${ }^{3-6}$ However, the specificity and function of HIV-1-specific mAbs produced by mucosal B-cell populations have yet to be explored,

\footnotetext{
${ }^{1}$ Human Vaccine Institute, Duke University Medical Center, Durham, North Carolina, USA and ${ }^{2}$ Department of Microbiology and Department of Mathematics \& Statistics, Boston University School of Medicine, Boston, Massachusetts, USA. Correspondence: SR Permar (sallie.permar@dm.duke.edu)

${ }^{3}$ The last two authors contributed equally to this work.
} 
primarily due to insufficient lymphocyte content of mucosal secretions and difficulties in obtaining mucosal tissue specimens. Importantly, colostrum is a rich source of mucosal B lymphocytes that are thought to originate in the gastrointestinal-associated lymphoid tissue and produce local antibodies that effectively protect infants against neonatal pathogens ("gut-mammary axis"). As a result, colostrum is an ideal source from which HIV-1 Env-specific antibodies can be isolated from mucosal B-cell populations, allowing characterization of the spectrum of HIV-1 Env-specific antibodies produced at mucosal surfaces.

Breastfeeding transmission accounts for over one-third of the 260,000 infant HIV-1 infections that occur annually. ${ }^{8,9}$ Remarkably, without anti-retroviral prophylaxis, only $10 \%$ of HIV-exposed breastfeeding infants become infected despite chronic mucosal HIV-1 exposure. ${ }^{10}$ This relatively low transmission rate suggests that the immunologic components in breast milk contribute to protecting the majority of infants exposed to HIV-1 via breastfeeding. Interestingly, despite high levels of secretory IgA in milk, ${ }^{7,11}$ the anti-HIV-1 humoral response seems to be primarily mediated by IgG antibodies. ${ }^{9,12,13}$ Prior work has further shown that HIV-1-specific antibodies with distinct anti-viral functions in milk may contribute to the low rate of virus transmission. ${ }^{13-15}$ In addition, we previously isolated two HIV-1-neutralizing IgG1 $\mathrm{mAbs}$ from colostrum, providing initial insight into the characteristics of Env-specific mAbs produced by colostrum B cells. ${ }^{16}$

We now describe the specificity, variable gene usage, and maturation of a large number of human mAbs isolated from colostrum from a cohort of $17 \mathrm{HIV}$-infected, lactating women. This study of colostrum HIV-1 Env-specific mucosal B-cell responses has identified characteristics of colostrum antibodies that suggest selective homing of IgG1-producing HIV-1 Env-specific B cells to the lactating mammary gland.

\section{RESULTS \\ Clinical characteristics of the cohort of 17 chronically HIV-1-infected, lactating Malawian women}

The median baseline CD4 + T-cell count of the chronically HIV-1-infected, lactating women included in this study was 275 cells per $\mu$ l (range, $80-592$ cells per $\mu \mathrm{l}$ ), and the median baseline plasma viral load was 5,207 RNA copies per ml (range, 200-174,976 RNA copies per ml) (Table 1). ${ }^{13}$ The median breast milk viral load at 4-6 weeks postpartum was 247 RNA copies per ml (range, 20-144,750 RNA copies per ml). Fifteen subjects were untreated during pregnancy with the exception of single-dose nevirapine during labor, and two began highly active anti-retroviral therapy during pregnancy. Three women transmitted HIV-1 to their infant via breastfeeding.

\section{High frequency of memory B cells in colostrum compared with blood}

The proportion of total CD19+ B cells ranged from $0.6 \%$ to $22 \%$ and $3 \%$ to $11 \%$ of total live cells isolated from colostrum and blood, respectively. Representative flow plots illustrating total B cell and Env-specific phenotype distribution of the colostrum and blood B-cell populations from one subject are shown in Figure 1. In both blood and colostrum, most B cells were IgD- memory, yet colostrum had a significantly higher proportion of IgD- memory B cells than blood (median: $96 \%$ vs. $62 \%$, respectively, $P<0.0001$, Wilcoxon matched-pairs signed rank test; Table 2). The proportions of CD27 + memory B cells (IgD-/CD27 + ) were similar in colostrum and blood (median: $34 \%$ vs. $28 \%$, respectively). Moreover, the median frequencies of plasmablasts and plasma cells $\left(\operatorname{IgD}-/ \mathrm{CD} 38^{\mathrm{Hi}}\right)$ were the same between colostrum and blood (both, 4\%). ${ }^{17,18}$ Colostrum and blood had similar median frequencies of B cells reactive with consensus $\mathrm{HIV}-1$ Env constructs, including a group M consensus (Con-S) gp120 (0\% and 0.1\%, respectively, $n=5)$ and gp140 ( $2 \%$ vs. $0.2 \%$, respectively; $n=12)$.

\section{HIV-1 Con-S gp140-specific sorting efficiently isolates HIV-1 Env-specific antibody-secreting B cells}

Colostrum-derived cell samples showed substantial variation in cell viability, necessitating optimization of the sort gating method. Initial samples were gated for total B cells (CD19+, CD3-/CD14-/CD16-/CD235a-) and total memory B cells (CD19+, CD3-/CD14-/CD16-/CD235a-, IgD-, CD38+), yielding few HIV-1 Env-specific mAbs defined by PCR amplification, transient transfection, and ELISA screening (two HIV-1 Env-specific mAbs isolated per 807 colostrum B cells amplified, $0.2 \%$ ). We then used antigen-specific sorting of $\mathrm{B}$ cells with HIV-1 Con-S gp120 with no increase in the yield of HIV-1 Env-specific antibodies from colostrum (1/409, 0.2\%). Finally, we used an HIV-1 Con-S gp140 probe for antigenspecific sorting to target gp41-reactive and conformationally dependent antibodies. This strategy increased the yield of HIV1 Env-reactive antibodies $(36 / 1,109,3 \%)$, showing that HIV-1 Con-S gp140 was more specific than Con-S gp120 for isolating $\mathrm{B}$ cells from colostrum. From blood-derived B cells, there was a $5 \%$ yield by ELISA of HIV Env-reactive antibodies sorted using the HIV-1 Con-S gp140 probe vs. 4\% from Con-S gp120specific sorting.

\section{Correlation between the proportion of HIV-1 Env-specific B cells in colostrum and HIV-1 Env-specific IgG response in whole breast milk}

The frequency of HIV-1 Env-specific colostrum B cells identified by antigen-specific flow cytometry (Con-S gp120, $n=5$ and Con-S gp140, $n=12$ ) was compared with plasma virus load during the third trimester of pregnancy and breast milk viral load at 4-6 weeks postpartum. No correlations were found between the colostrum Env-specific B-cell frequency and plasma or milk viral load (Figure 2a,b). Furthermore, no correlation was observed between the proportion of HIV-1 Env-specific B cells and the consensus Con-6 gp120-binding $(R=0.41 ; P=0.15)$ or Con-S gp140-binding $\quad(\mathrm{R}=0.49$; $P=0.08$ ) IgG response in milk (both, Spearman rank correlation; Figure 2c,d). With B cells isolated by gp120specific sorting excluded, the colostrum Env-specific B-cell frequency and the gp41-binding IgG response in milk were not associated (Figure 2e). Similarly, there was no correlation 
Table 1 Clinical characteristics and mAbs isolated from blood (BLD) and colostrum (CLM) of 17 chronically HIV-1-infected lactating women from Malawi (CHAVI009)

\begin{tabular}{|c|c|c|c|c|c|c|c|}
\hline $\begin{array}{l}\text { Subject } \\
\text { ID }\end{array}$ & $\begin{array}{c}\text { Peripheral CD4 }+ \\
\text { T-cell count }^{\mathrm{a}}\end{array}$ & $\begin{array}{c}\text { Plasma } \\
\text { viral load }\end{array}$ & $\begin{array}{l}\text { Breast milk } \\
\text { viral load }^{\mathrm{b}}\end{array}$ & Treatment & $\begin{array}{l}\text { BLD B-cell mAbs } \\
\text { (\% Env-reactive) }\end{array}$ & $\begin{array}{l}\text { CLM B-cell mAbs } \\
\text { (\% Env-reactive) }\end{array}$ & B-cell sort method \\
\hline $\mathrm{CH} 0608$ & 275 & 200 & 20 & HAART ${ }^{\mathrm{C}}$ & $24(4 \%)$ & 0 & Total B, Con-S gp120 \\
\hline $\mathrm{CH} 4403^{f}$ & 208 & 100,892 & 144,750 & sdNVP & 0 & $1(0 \%)$ & Con-S gp120, Con-S gp140 \\
\hline $\mathrm{CH} 8700$ & 123 & 200 & 285 & HAART & $14(0 \%)$ & 0 & Con-S gp140 \\
\hline $\mathrm{CH} 8908$ & 178 & 9,246 & 120 & sdNVP & 0 & $1(100 \%)$ & Con-S gp140 \\
\hline $\mathrm{CH} 9105$ & 266 & 5,207 & 120 & sdNVP & $12(83 \%)$ & $14(100 \%)$ & Con-S gp140 \\
\hline CH9208 & 447 & 200 & 120 & sdNVP & $6(33 \%)$ & $8(100 \%)$ & Con-S gp140 \\
\hline $\mathrm{CH} 9300$ & 415 & 91,717 & 254 & sdNVP & 35 (23\%) & 5 (40\%) & Con-S gp120, Con-S gp140 \\
\hline $\mathrm{CH} 0009$ & 429 & 1,256 & 120 & sdNVP & $6(83 \%)$ & $2(50 \%)$ & Con-S gp140 \\
\hline $\mathrm{CH} 0305$ & 198 & 200 & 240 & sdNVP & $3(100 \%)$ & 0 & Con-S gp140 \\
\hline $\mathrm{CHO} 404^{f}$ & 80 & $22,600^{b}$ & 29,425 & sdNVP & 19 (58\%) & $10(70 \%)$ & Con-S gp140 \\
\hline $\mathrm{CH} 0301$ & 406 & 75,934 & 790 & sdNVP & $6(0 \%)$ & $3(0 \%)$ & Total B \\
\hline $\mathrm{CH}_{1209^{f}}$ & 153 & 174,976 & 26,818 & sdNVP & $14(7 \%)$ & $2(0 \%)$ & Total B \\
\hline
\end{tabular}

Env, envelope; mAbs, monoclonal antibodies.

${ }^{a}$ Measured during third trimester of pregnancy.

bMeasured 4-6 weeks postpartum.

'Subject received Highly Active Anti-Retroviral Therapy (HAART) during pregnancy and breastfeeding

${ }^{\mathrm{d}}$ Four- to six-week milk sample unavailable.

e Subject and infant received single-dose Nevirapine (sdNVP) at delivery.

f Subject transmitted HIV-1 postnatally to infant.

between the colostrum Env-specific B-cell frequency and milk neutralization response against a tier 1 neutralization-sensitive clade-matched virus (C.MW965; Figure 2f).

\section{IgG1 predominance of HIV-1 Env-reactive mAbs isolated from colostrum B cells}

From blood samples of 17 women, we isolated 1,495 singlesorted B cells, of which 243 yielded a pair of $\mathrm{V}_{\mathrm{H}} \mathrm{DJ} \mathrm{H}_{\mathrm{H}}$ and $\mathrm{V}_{\mathrm{L}} \mathrm{J}_{\mathrm{L}}$ genes. In all, 70 of these antibodies were excluded from analysis due to duplicate or indeterminate heavy chain sequences, and 51 of the 173 remaining blood antibodies were Env reactive by ELISA (29\%). We isolated 2,919 colostrum B cells from the same cohort, yielding 161 antibodies, of which 80 were excluded as above, and 39 of the remaining 81 colostrum antibodies were HIV-1 Env reactive by ELISA (48\%).

Non-reactive antibodies produced by blood B cells were primarily IgM isotype (71\%), while most non-reactive antibodies in colostrum were IgG1 isotype (55\%; Figure 3). A small number of IgA (9\%) and IgD (2\%) non-reactive antibodies were isolated from blood B cells. Similarly, we found few colostrum B cells producing IgA antibodies (7\%), and none were HIV-1 Env specific. Interestingly, Env-reactive antibodies in colostrum were all IgG1 isotype (100\%), consistent with our prior isolation of only IgG1 HIV-1 Env-specific colostrum antibodies. ${ }^{16}$ In contrast, IgA, IgG1, IgG3, IgD, and IgM Env-reactive antibodies were isolated from blood.

\section{Restricted $V_{\mathrm{H}}$ gene usage in HIV-1 Env-reactive mAbs produced by colostrum $B$ cells}

$\mathrm{mAbs}$ isolated from blood and colostrum used a total of 30 and 27 different $V_{H}$ gene subfamilies, respectively (Figure 4). The identified HIV-1 Env-specific blood and colostrum antibodies used 20 and 12 different $V_{H}$ gene subfamilies, respectively. HIV-1 Env-specific antibodies from both compartments employed $V_{H}$ $1 \sim 2,1 \sim 69,3 \sim 15,3 \sim 23,3 \sim 30,3^{\sim} 30-3,3 \sim 49$, and $5^{\sim} 51$ alleles. The only identified unique $V_{\mathrm{H}}$ gene subfamily used by Envreactive colostrum B-cell antibodies was $3 \sim 43$. Notably, $V_{H}$ $1 \sim 69$ constituted $49 \%$ of the gene usage of HIV-1 Env-specific colostrum antibodies, compared with $12 \%$ in non-reactive colostrum antibodies ( $P=0.0005$, Fisher's exact test). Similarly, $\mathrm{V}_{\mathrm{H}} 1^{\sim} 69$ was the most commonly used gene subfamily in HIV-1 Env-specific antibodies from blood (20\%), significantly higher than in non-reactive blood antibodies $(6 \%, P=0.01$, Fisher's exact test). Yet, $V_{\mathrm{H}} 1 \sim 69$ was used at a significantly higher rate in HIV-1 Env-specific antibodies isolated from colostrum than blood ( $P=0.006$, Fisher's exact test). 

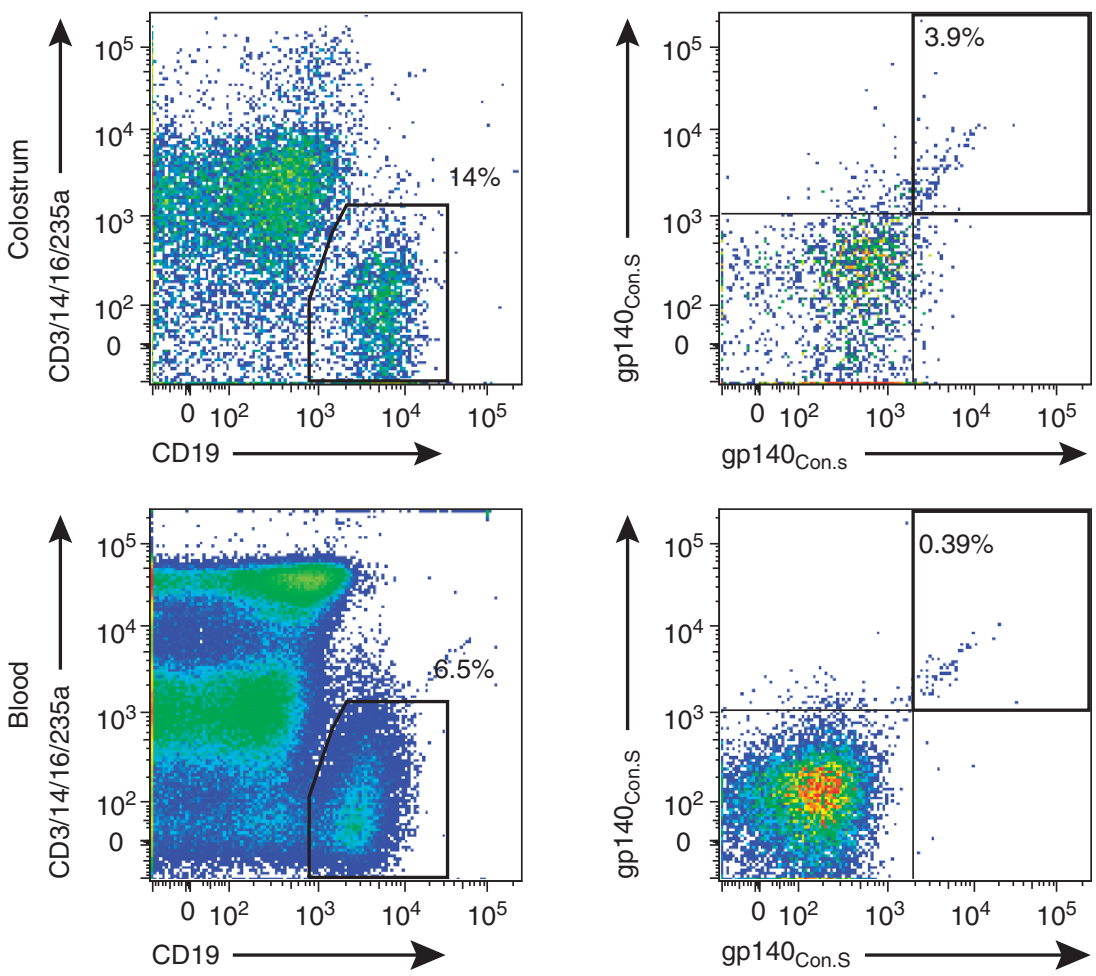

Figure 1 Total B cell and Con-S gp140 HIV-1 envelope (Env)-specific B-cell populations in colostrum (top) and blood (bottom) of an HIV-1-infected, lactating woman. Total B cells were gated as CD3-/CD14-/CD16-/CD235a- and CD19 + (left). HIV-1 Env-specific B cells were gated from the total B-cell population as Con-S gp140 AF647 + /Con-S gp140 BV421 + (right).

Table 2 Colostrum and blood B lymphocyte phenotype

\begin{tabular}{|c|c|c|c|c|c|}
\hline & $\begin{array}{c}\% \text { IgD- (range) } \\
\quad(n=17)\end{array}$ & $\begin{array}{l}\% \text { IgD-/CD27+ } \\
\text { (range) }(n=17)\end{array}$ & $\begin{array}{l}\% \text { lgD-/CD38 } \\
\text { (range) }(n=17)\end{array}$ & $\begin{array}{c}\% \text { IgD-/Con-S gp120 }++ \\
\text { (range) }(n=5)\end{array}$ & $\begin{array}{c}\% \text { IgD-/Con-S gp140 }++ \\
\text { (range) }(n=12)\end{array}$ \\
\hline Colostrum & $96^{a}(69-100)$ & $34(0-83)$ & $4(0-39)$ & $0(0-4.0)$ & $2(0.2-6)$ \\
\hline$P$-value ${ }^{b}$ & $<0.0001$ & 0.13 & 0.33 & 0.38 & 0.06 \\
\hline
\end{tabular}

a Median.

bilcoxon matched-pairs signed rank test comparing colostrum and blood; $P<0.05$ in bold.

\section{CDR3 lengths and somatic hypermutation frequencies of colostrum HIV-1 Env-reactive IgG B-cell mAbs are similar to those isolated from peripheral B cells}

As broadly HIV-1-neutralizing antibodies often display long CDR3 lengths and high somatic hypermutation rates ${ }^{19}$ we examined these parameters in blood and colostrum Env-specific mAbs. The mean CDR3 lengths of non-reactive IgG antibodies in blood and colostrum were similar (15 and 17 amino acids, respectively), as were the mean CDR3 lengths of HIV-1 Env-reactive IgG antibodies in blood and colostrum (16 and 17 amino acids, respectively; Table 3). Somatic hypermutation rates were also similar between Env-reactive IgG antibodies from blood (6\%) and colostrum (7\%), as well as between nonreactive blood and colostrum antibodies (both, 6\%).
High rate of gp120-directed IgG1 antibodies in the colostrum HIV-1 Env-specific B-cell mAb repertoire

The majority of the neutralizing epitopes identified on the HIV-1 Env protein complex are contained within the gp120 protein ${ }^{20}$ whereas the gp41 protein has only a single identified neutralizing site, the membrane proximal external region. ${ }^{21}$ However, the predominant early target of acute HIV-1 Env antibody responses in plasma and the genital mucosa is gp41. ${ }^{22-24}$ Therefore, we compared the distribution of gp120and gp41-directed antibodies produced by blood and colostrum B cells. Strikingly, while the majority of HIV-1 Env-reactive antibodies isolated from blood were gp41 specific (65\%), only $21 \%$ of the colostrum HIV-1 Env-reactive antibodies were gp41 directed $(P<0.0001$, Fisher's exact test; 

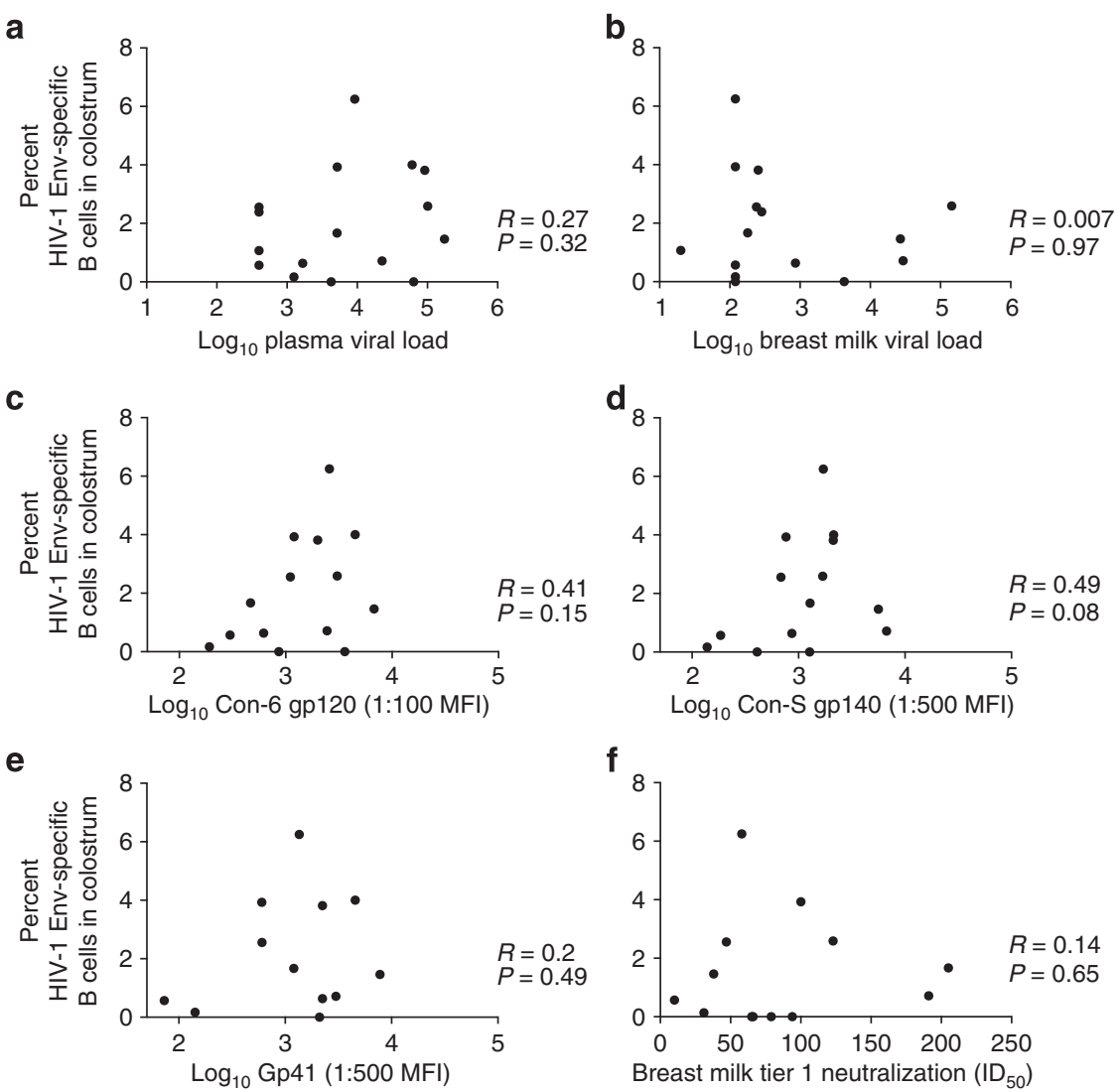

Figure 2 Correlation of the proportion of HIV-1 envelope (Env)-specific B cells isolated from colostrum with virus load and humoral responses in milk. There is no association between the proportion of HIV-1 Env-specific B cells in colostrum and (a) plasma viral load during pregnancy or (b) breast milk viral load at 4-6 weeks postpartum. There is no correlation between the proportion of HIV-1 Env-specific B cells in colostrum and (c) breast milk HIV-1 Envspecific Con-6 gp120-binding $(R=0.41, P=0.15)$ or (d) Con-S gp140-binding IgG response $(R=0.49, P=0.08$; both, Spearman Rank Correlation). The proportion of HIV-1 Env-specific B cells in colostrum is not correlated with (e) gp41-binding IgG response or (f) breast milk tier 1 HIV-1 (C.MW956) neutralization potency. MFI, mean fluorescence intensity.
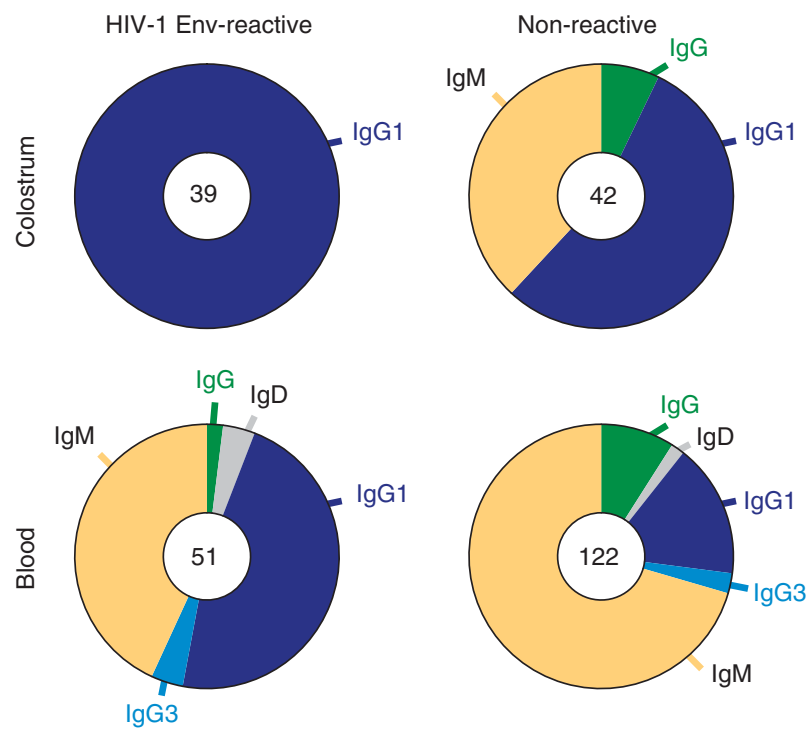

Figure 3 HIV-1 envelope (Env)-reactive monoclonal antibodies isolated from colostrum are IgG1 isotype. The isotype distribution of HIV-1 Envreactive (left) and non-reactive (right) antibodies isolated from colostrum (top) and blood (bottom) of chronically HIV-1-infected, lactating women is shown by color. The number of antibodies in each population is indicated in the center of each donut.
Table 4). Rather, more HIV-1 Env-reactive antibodies isolated from colostrum were gp 120 specific than in blood (44\% vs. $16 \%$, respectively, $P=0.005$, Fisher's exact test). In addition, significantly more colostrum than blood antibodies were multi-Env specific (bind gp120 and gp 41 antigens; $33 \%$ vs. $12 \%$, respectively, $P=0.02$, Fisher's exact test). If only those antibodies produced by Con-S gp140-specific-sorted B cells were analyzed, differences in antigen specificity between Env-reactive colostrum and blood antibodies persisted (Table 4). Finally, gp120-specific and gp41-specific antibodies isolated from colostrum used $V_{\mathrm{H}} 1^{\sim} 69$ gene at a relatively higher frequency than those in blood (59\% vs. $25 \%$ for gp120 and $50 \%$ vs. $18 \%$ for gp 41 ).

\section{Non-reactive and gp41-directed B-cell mAbs of HIV-1-infected, lactating mothers who transmitted HIV-1 postnatally to their infant}

We aimed next to compare the HIV-1 Env-specific colostrum B-cell repertoire of the postnatally transmitting and nontransmitting women included in this cohort, though our analysis was limited by the low number of transmitting women (three) and low colostrum antibody recovery from this group. The women who transmitted HIV-1 to their infant via 

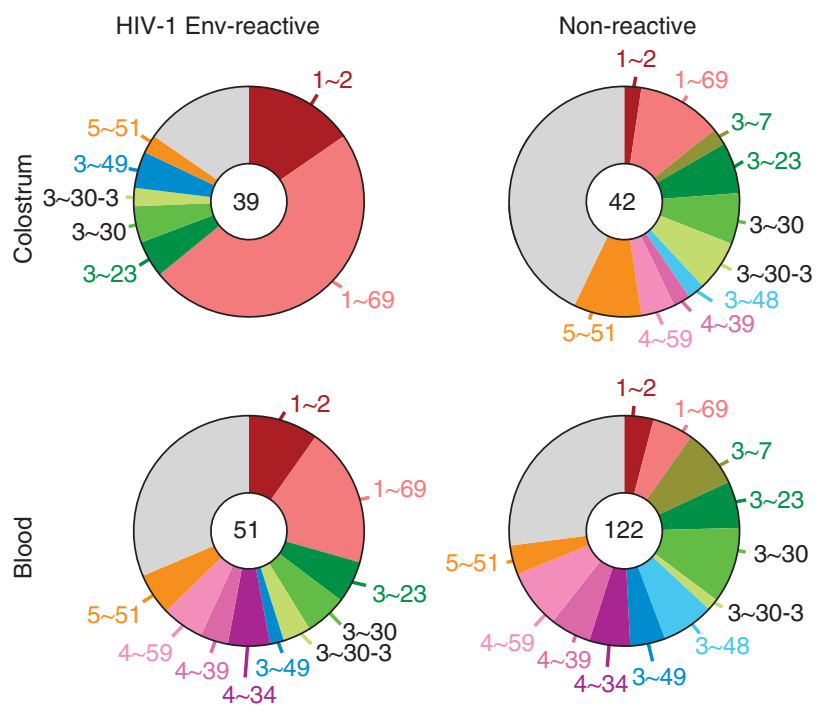

Figure 4 Distinct $\mathrm{V}_{\mathrm{H}}$ gene usage in HIV-1 envelope (Env)-reactive antibodies isolated from colostrum. These charts show the $V_{H}$ gene usage in HIV-1 Env-reactive (left) and non-reactive (right) antibodies isolated from colostrum (top) and blood (bottom). The number of antibodies in each population is indicated in the center of each donut. Subfamilies contributing at least $6 \%$ of the total $\mathrm{V}_{\mathrm{H}}$ gene usage in one antibody population are shown on all donuts in color. The $\mathrm{V}_{\mathrm{H}} 1 \sim 69$ gene subfamily was used at a significantly higher rate in HIV-1 Env-specific antibodies from colostrum compared with those from blood $(P=0.006$, Fisher's exact test).

Table 3 Somatic hypermutation frequencies and CDR3 lengths are not significantly different between HIV-1 Env-reactive IgG antibodies in blood and colostrum

\begin{tabular}{lrcc}
\hline & $\begin{array}{c}\text { Non- } \\
\text { reactive }\end{array}$ & $\begin{array}{c}\text { HIV-1 } \\
\text { Env-reactive }\end{array}$ & P-value \\
\hline Mean CDR3 length (amino acids) & 15 & 16 & 0.83 \\
Blood & 17 & 17 & 0.94 \\
Colostrum & 0.12 & 0.13 & \\
$\begin{array}{l}\text { P-value } \\
\text { a }\end{array}$ & & & \\
Mean mutation frequency (\%) & & & \\
Blood & 6 & 6 & 0.97 \\
Colostrum & 6 & 7 & 0.24 \\
P-value & 0.90 & 0.21 & \\
\hline
\end{tabular}

CDR3, complementarity-determining region 3; Env, envelope.

${ }^{a}$ Analyses performed using linear mixed model; $P>0.05$ is not significant.

breastfeeding had either only non-reactive colostrum B-cell antibodies isolated (CH4403, $n=1 ; \mathrm{CH} 1209, n=2)$ or predominately gp41-specific colostrum B-cell antibodies (CH0404, $n=10: 50 \%$ gp41 specific, $10 \%$ gp120 specific, $10 \%$ multi-Env specific, 30\% non-reactive; Supplementary Figure S2 online). Although colostrum B cells of transmitting subject CH0404 produced predominately gp41-specific antibodies, this subject's blood had equal numbers of gp41- and gp120-specific antibodies (both, $n=5$ ). Of the 14 subjects who did not transmit HIV-1 to their infant postnatally, one or more HIV-1 Env-reactive colostrum antibodies were isolated from seven subjects. Their HIV-1 Env-reactive colostrum B-cell antibody populations consisted primarily of gp120-binding antibodies, with the exception of CH9300 (gp140, $n=1$; gp120, $n=1)$ and CH9803 (gp41, $n=2$; Supplementary Figure S2).

\section{Cross-compartment clonal IgG1-secreting B-cell lineage identified}

Six groups of clonally related HIV-1 Env-reactive B-cell antibodies were identified in blood and/or milk of three HIV-1infected subjects: CH9105, CH9208, and CH0404 (Table 5). In addition, one cross-compartment clonal set was identified which contained two gp120-specific colostrum antibodies and one gp120-specific blood antibody (Figure 5), indicating systemic trafficking of colostrum HIV-1 Env-specific B cells. Like the Env-reactive antibodies isolated from colostrum, all clonally related antibodies were IgG1 isotype. This finding was surprising given the previously reported prevalence of IgA-secreting cells in colostrum ${ }^{7,11,25}$ and suggests that class switching to $\operatorname{IgA}$ is not required for $\mathrm{B}$ cells to home to the lactating mammary gland.

\section{DISCUSSION}

Breastfeeding remains a significant mode of HIV-1 transmission in many developing countries, but the protective role of HIV-1 Env-specific maternal antibodies has not been consistently established. ${ }^{8,9}$ Several studies have demonstrated a predominately IgG anti-HIV-1 Env response in breast milk, ${ }^{9,12,13}$ despite the high concentration of total IgA in breast milk and other mucosal compartments. ${ }^{7,11}$ Furthermore, the HIV-1 Env-specific IgG responses in breast milk correlate with those in blood, albeit at a two log lower magnitude, suggesting that IgG transudate from plasma to the breast milk compartment is the predominant breast milk anti-HIV-1 antibody response. ${ }^{13}$ Yet, there is also some evidence of distinct anti-HIV-1 IgG antibody specificities and functions between the two compartments. ${ }^{15}$ Moreover, antibody-dependent cellular cytotoxicity response of breast milk HIV-1 Env-specific IgG was recently associated with protection against postnatal HIV-1 transmission. ${ }^{14}$ Thus, our study provides a characterization of the genetic qualities and specificity of breast milk HIV-1 Env-specific B cells, facilitating better strategies to target an effective local antibody response through maternal vaccination.

Maternal B cells in breast milk, specifically IgA-secreting and potentially IgG-secreting B cells, appear to home to the lactating mammary gland from mesenteric lymph nodes in gastrointestinal-associated lymphoid tissue along the "gut-mammary axis," and many are specific for gut microflora. ${ }^{7,26-28}$ Because the lymphocyte content is high in colostrum compared with mature milk, ${ }^{10}$ this fluid provided a unique opportunity to examine the HIV-1 Env-specific B cells that traffic to this mucosal compartment. In our B-cell repertoire analysis, we observed that the colostrum of chronically HIV-1-infected women contained a significantly greater proportion of memory 
Table 4 Predominance of gp120-directed HIV-1 Env-specific antibodies isolated from colostrum B cells

\begin{tabular}{|c|c|c|c|c|}
\hline & gp120 & gp41 & gp140 & Multi-Env \\
\hline Blood $(n=51)$ & $8(16 \%)$ & 33 (65\%) & $4(8 \%)$ & $6(12 \%)$ \\
\hline Colostrum $(n=39)$ & 17 (44\%) & 8 (21\%) & $1(3 \%)$ & $13(33 \%)$ \\
\hline $\mathrm{V}_{\mathrm{H}} 1 \sim 69$ usage & $10(59 \%)$ & $4(50 \%)$ & 0 & $5(38 \%)$ \\
\hline Blood $(n=40)$ & $8(20 \%)$ & 24 (60\%) & $4(10 \%)$ & $4(10 \%)$ \\
\hline$V_{H} 1 \sim 69$ usage & $2(25 \%)$ & $4(17 \%)$ & 0 & $1(25 \%)$ \\
\hline Colostrum $(n=36)$ & $14(39 \%)$ & 8 (22\%) & $1(3 \%)$ & $13(36 \%)$ \\
\hline$V_{H} 1^{\sim} 69$ usage & $9(64 \%)$ & $4(50 \%)$ & 0 & $5(38 \%)$ \\
\hline
\end{tabular}

Env, envelope; mAbs, monoclonal antibodies.

Table 5 Single and cross-compartment HIV-1 Env-reactive clonal B-cell lineages isolated from blood (BLD) and colostrum (CLM)

\begin{tabular}{|c|c|c|c|c|c|}
\hline Clonal lineage $^{a}$ & Compartment (number of mAbs) & Env specificity (number of mAbs) & Ig isotype & $\mathrm{V}_{\mathrm{H}}$ & $\mathrm{V}_{\mathrm{K}}$ or $\mathrm{V}_{\mathrm{L}}$ \\
\hline $\mathrm{DH} 279, \mathrm{DH} 280$ & CLM (2) & gp120 (2) & lgG1 & $1 \sim 69$ & $1 \sim 12$ \\
\hline DH276, DH277, DH278 & CLM (2)/BLD (1) & gp120 (3) & $\operatorname{lgG1}$ & $1 \sim 69$ & $3 \sim 20$ \\
\hline \multicolumn{6}{|l|}{ CH9208 } \\
\hline DH285, DH286, DH287 & CLM (3) & gp120 (3) & $\lg \mathrm{G} 1$ & $1 \sim 69$ & $3 \sim 20$ \\
\hline \multicolumn{6}{|l|}{$\mathrm{CHO4O4}$} \\
\hline $\mathrm{DH} 288, \mathrm{DH} 289$ & CLM (2) & gp41 (2) & $\operatorname{lgG1}$ & $1 \sim 69$ & $3^{\sim 11}$ \\
\hline
\end{tabular}

Env, envelope; mAbs, monoclonal antibodies.

The clonal lineages are named by their member antibody numbers.

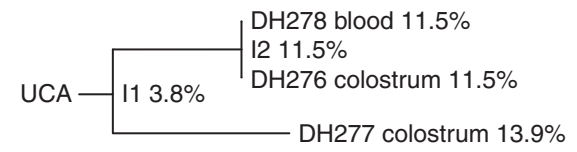

$$
0.05
$$

Figure 5 Cross-compartment gp120-specific, IgG1-secreting B-cell clonal lineage tree from subject $\mathrm{CH} 9105$. One cross-compartment clonal set containing two gp120-specific colostrum antibodies and one blood antibody is illustrated. The inferred unmutated common ancestors (UCAs) are at the roots, the intermediates are at the branches (I1 and I2), and the antibody heavy chain sequences are at the leaves. The legend indicates the scale of evolutionary distance. All clones are reactive to Con-S gp120 by ELISA, and mutation frequencies of intermediate and antibody sequences relative to the inferred UCA are shown as percentages.

B cells than blood $(P<0.0001)$, corresponding with previous data showing that breast milk B cells are primarily classswitched memory B cells. ${ }^{12}$ Importantly, the HIV-1 Envspecific colostrum antibodies had reduced isotype breadth compared with those from blood, as all were IgG1 isotype, consistent with a previous report of predominately IgG1producing HIV-1 Env-specific B cells in breast milk. ${ }^{12}$ Furthermore, our identification of an HIV-1 Env-reactive cross-compartment clonal IgG1-secreting B-cell lineage indicates systemic trafficking of IgG1-producing B cells to the lactating mammary gland.

The absence of a correlation between plasma or milk virus load and the colostrum HIV-1 Env-specific B-cell frequency in this study was unsurprising given the evidence that systemic virus populations continually repopulate the breast milk compartment virus pool. ${ }^{29}$ Furthermore, the administration of nevirapine at delivery likely interrupted any relationship between the local B-cell responses and milk viral load. Prior studies have demonstrated that only low levels of virus neutralization responses are detectable in breast milk, and these whole milk responses did not correlate with breast milk viral load. ${ }^{13}$ The lack of correlation between the proportion of HIV-1 Env-specific B cells and HIV-1 Env-specific IgG binding responses or viral neutralization in milk signifies that a locally generated colostrum B-cell IgG1 antibody response may only 
contribute to a small portion of the total milk anti-HIV-1 IgG response. Importantly, however, locally produced antibodies may be able to interfere with transmission of breast milk virus within the infant gastrointestinal tract. The predominance of IgG1-producing antigen-specific B cells in colostrum also has important implications for the traditional paradigm that chiefly IgA-secreting B cells are trafficked within the gut-mammary axis. ${ }^{7}$

While there is a strong correlation between the total HIV-1 Env-specific IgG responses in blood and milk, the HIV-1 Env-specific IgA responses in milk and plasma are not associated. ${ }^{13} \mathrm{~A}$ detectable HIV-1-specific IgA response in milk of HIV-1-infected women has been demonstrated at 15 days postpartum in fewer individuals and at lower magnitude compared with the milk HIV-1 Env-specific IgG response. ${ }^{9}$ In our colostrum B-cell analysis, the few IgA colostrum antibodies isolated were non-reactive. Our findings lend support to the idea that HIV-1 Env-specific IgA-secreting B cells may be primarily located within mammary tissue, not in the secretory fluid. HIV-1 Env-specific IgA-producing B cells have not been easily isolated from mucosal compartments; thus, it remains unclear whether mucosal IgA responses have a role in protecting the mucosal surface against HIV-1 infection.

The HIV-1 Env-specific mAbs isolated from colostrum in our study did not have a particularly high mean somatic hypermutation rate or long CDR3 length, characteristics of broadly neutralizing antibodies isolated from blood. $^{3-6,19,21,30,31}$ However, colostrum and blood HIV-1 Env-specific B cell $m A b s$ were distinguishable by different $\mathrm{V}_{\mathrm{H}}$ gene usage. HIV-1 Env-reactive B-cell colostrum mAbs demonstrated a higher rate of $\mathrm{V}_{\mathrm{H}} 1 \sim 69$ usage than non-reactive colostrum antibodies or HIV-1 Env-reactive B-cell blood mAbs $(P=0.0005$ and $P=0.006$, respectively, Fisher's exact test). Previous data have suggested that $\mathrm{V}_{\mathrm{H}} \sim 69$ gene usage may facilitate direct heavy chain CDR2 interaction with the CD4-inducible epitope on gp120. ${ }^{30}$ Therefore, the higher frequency of $\mathrm{V}_{\mathrm{H}} 1^{\sim} 69$ gene subfamily usage may relate to the distinct specificities of Env-specific antibodies in colostrum compared with blood. Conversely, the antigen-specific sorting employed in this study could have skewed gene usage by better targeting specific B-cell populations. However, both gp41-specific and gp120-specific colostrum antibodies used $\mathrm{V}_{\mathrm{H}} 1^{\sim} 69$ at a somewhat higher frequency than those from blood, indicating that high $\mathrm{V}_{\mathrm{H}} 1 \sim 69$ usage by colostrum $\mathrm{B}$ cells may not be solely driven by antigen binding properties.

The proportions of gp120-, multi-Env-, and gp41-specific antibodies isolated from the systemic and mucosal compartments in this study are not biologically representative, as our use of antigen-specific B-cell sorting biased the results by selecting for HIV-1 Env-specific antibodies that bind to the antigen employed. Given the small numbers of colostrum Env-specific B cells, slight differences in the affinity of the gp140 vs. gp120 protein for the B-cell receptor may explain the superior ability of the gp140 epitope to isolate antigen-specific B cells. However, as Con-S gp140-specific sorting is effective in isolating both gp41-specific and gp120-specific antibodies, it is an unlikely source of bias toward one specificity. ${ }^{22}$ Our data therefore indicate that gp120-binding $\mathrm{mAbs}$ are more frequently produced by colostrum than by blood HIV-1 Env-specific B cells, and whether they are more common in chronically HIV-infected lactating women who do not transmit HIV-1 to their infant postnatally should be studied in more detail. As there were only three postnatally transmitting women in the entire CHAVI009 cohort, all with low colostrum mAb recovery, an association between gp120-specific B cell predominance in colostrum and no postnatal transmission of HIV-1 cannot be concluded with our current panel of antibodies, yet highlights the need for further investigation.

A primarily gp 120- and multi-Env-specific B-cell response in the lactating mammary gland contrasts with the gp41-specific antibody predominance of the acute systemic and mucosal B-cell response to HIV-1. ${ }^{22,24}$ The mechanism of distinct antigen-specific $\mathrm{B}$ cell homing to the mammary gland is unclear but could be hormonally or receptor mediated. ${ }^{7,26,32} \mathrm{Gp} 120$ directed antibodies may have more neutralizing capability than gp41-directed antibodies, as neutralizing gp41-directed antibodies are rare and have only been found to target a single epitope (membrane proximal external region). Selective trafficking of HIV-1 Env-specific B cells with distinct $V_{H}$ gene usage and high rates of gp120-binding antibodies to the lactating mammary gland could thus produce a more effective antigen-specific response that contributes to the protection of the majority of HIV-exposed breastfed infants against postnatal HIV-1 transmission. Moreover, this distinct B-cell population in the colostrum compartment is a potential target of future maternal vaccine candidates developed to reduce postnatal HIV-1 transmission.

\section{METHODS}

Ethics statement. This study (CHAVI009) was approved by the Division of DAIDS; NIAID; NIH; the College of Medicine Research and Ethics Committee in Malawi (P.06/06/440); and the institutional review board at Duke University (Pro00030437). Subjects signed a written consent.

Subjects and colostrum processing. Colostrum and blood samples were obtained from a total of 77 chronically HIV-1-infected, lactating women enrolled between 2008 and 2009 in the CHAVI009 study in Malawi, 17 of which were included in this study based on high colostrum cell number to maximize yield of antigen-specific B cells. ${ }^{13,29}$ The subjects were followed throughout pregnancy and breastfeeding. All subjects and infants received a single dose of nevirapine during the peripartum period. Infant HIV-1 status was determined by whole blood HIV-1 DNA PCR. ${ }^{33}$ Postnatal HIV-1 transmission was defined by negative infant whole blood HIV-1 DNA PCR at birth and at 4-6 weeks of age, followed by a positive whole blood HIV-1 DNA PCR at 3 months of age or later. ${ }^{33}$

Baseline plasma viral load and peripheral CD4 T-cell count were measured during the subjects' third trimester of pregnancy. Breast milk and blood samples were collected from the subjects within 1 week following delivery and again at 4-6 weeks postpartum. Breast milk collected within 7 days of delivery was defined as "colostrum." Breast milk viral load was measured at 4-6 weeks postpartum due to the limited amount of colostrum able to be collected. The milk samples were processed within $4 \mathrm{~h}$ of collection and separated into cellular and skim milk fractions by low speed centrifugation as previously 
described. ${ }^{34}$ Colostrum (CLM) cells and peripheral blood mononuclear cells (PBMC) were then cryopreserved within $6 \mathrm{~h}$ of collection and stored viably in liquid nitrogen.

Flow cytometry panel antibodies and isolation of B cells from colostrum and blood. CLM and PBMC isolated from EDTAanticoagulated blood were thawed in a $37^{\circ} \mathrm{C}$ water bath. Right and left breast CLM were combined if both available. The samples were then immediately resuspended in $14 \mathrm{ml}$ pre-warmed RPMI/10\% FBS and afterward centrifuged at $250 \times g$ for $10 \mathrm{~min}$. Following removal of the supernatant, the cells were resuspended and washed in R10, once to remove residual DMSO from the freezing media and a second time for cell counting. After a count was obtained, the cells were washed and resuspended with $\mathrm{PBS} / 2 \%$ FBS before labeled antibody staining.

Thawed CLM and PBMC were stained with the following panel of antibodies for flow cytometry: anti-IgM-fluorescein isothiocyanate (G20-127; BD Biosciences, San Jose, CA), anti-IgD-phycoerythrin (PE) (IA6-2; BD Biosciences), anti-CD10-electron coupled dye (ALB1; BD Biosciences), anti-CD3-PE-Cy5 (HIT3a; BD Biosciences), antiCD14-PE-Cy5 (RMO52; Beckman Coulter, Brea, CA), anti-CD16PE-Cy5 (3G8; BD Biosciences), anti-CD235a-PE-Cy5 (GA-R2; BD Biosciences), anti-CD27-PE-Cy7 (323; eBiosciences, San Diego, CA), anti-CD38- allophycocyanin -Alexa Fluor 700 (LS198-4-3; Beckman Coulter), and anti-CD19-allophycocyanin-Cy7 (SJ25C1; BD Biosciences). CLM and PBMC were stained with either HIV-1 consensus clade C gp120 Env (Con-S gp120) or HIV-1 group M consensus gp140 Env (Con-S gp140) for antigen-specific B-cell sorts. Con-S gp120 was labeled with Pacific Blue (PacBlue) and AF647 (both from Invitrogen, Carlsbad, CA). The Con-S gp140 protein included an AviTag that was then biotinylated via biotin ligase. The biotinylated protein was then tetramerized to Streptavidin-BV421 (Sony Biotechnology, Champaign, IL) and Streptavidin-AF647 (Invitrogen). Aqua Vital Dye was used to distinguish live from dead cells (Invitrogen). Controls consisted of matching isotype antibodies.

$\mathrm{B}$ cells from blood and colostrum were isolated and sorted according to four methods. Total B cells were gated as viable (Aqua Vital Dye -), CD3-/CD14-/CD16-/CD235a-, and CD19+ . Total memory B cells were further selected by gating as IgD- and CD38 + from the total B-cell population. For antigen-specific sorts, total B cells were gated as Con-S PacBlue + and Con-S AF647 + (Con-S gp120 specific), or Con-S BV421 + and Con-S AF647 + (Con-S gp140 specific). The B cells were individually sorted into 96-well plates pre-charged with an RNA stabilization cocktail and cryopreserved as described previously. ${ }^{6,35}$

Phenotyping of isolated B cells. Flow cytometry data were collected on the FACSAria2 instrument with the FACSDiVa software (BD Biosciences, San Jose, CA) and analyzed by manual gating with the FlowJo software (Tree Star Inc., Ashland, OR). The complete gating strategy for the B-cell phenotype analysis is shown in Supplementary Figure S1A. A broad lymphocyte gate was used to incorporate larger plasma cells and activated B cells which may be somewhat granular, as well as those associated with fat globules in colostrum due to its high lipid content. ${ }^{17,26}$ Side scatter and forward scatter width vs. height geometric gating excluded doublets and clumps. All Aqua Vital Dye + (dead) cells were omitted, and the total B-cell population was defined as CD3-/CD14-/CD16-/CD235a- and CD19+ live cells. This gate was determined for the blood B-cell population of each subject and copied to the colostrum B-cell population. B-cell phenotype was further identified as IgD- (memory and antibody-secreting B cells), IgD-/ $\mathrm{CD} 27+$ (class-switched memory B cells), IgD-/CD $38^{\mathrm{Hi}}$ (plasmablasts or plasma cells), and Con-S gp120 or Con-S gp140 specific. $^{17}$

To ensure that inadequate compensation was not responsible for the observed diagonal of antigen-specific B cells, the compensation matrix was artificially adjusted to increase the compensation for each antigen-specific reagent fluorochrome (Supplementary Figure S1B). Additionally, we stained PBMC and colostrum cells from HIV-1 uninfected women to determine whether there was a background level of staining with the reagents; no staining of memory B cells was observed in these samples (Supplementary Figure S1C).

Ig gene amplification, sequencing, and screening for HIV-1 Env reactivity. After colostrum B-cell sorting, the expressed Ig $V_{H}$ and variable light chain $\left(\mathrm{V}_{\mathrm{L}}\right)$ genes were amplified by reverse transcription and nested PCR as previously described. ${ }^{35,36}$ The PCR products were purified and sequenced. ${ }^{35}$ Ig isotype was determined by sequence homology, and somatic hypermutation frequencies, inferred V(D)J rearrangement, and CDR3 length were determined by Somatic Diversification Analysis, a web-based programming algorithm. ${ }^{37}$ Overlapping PCR was performed to co-express the variable region PCR products with full-length IgG1 (for heavy chain) and kappa or lambda (for light chain) cassettes, and the PCR products were transiently transfected in 293T cells without the need for a cloning step as described previously. ${ }^{23,35,36}$ The supernatants of transfected cells were screened for reactivity against the following HIV-1 Env proteins by enzyme-linked immunoassay (ELISA): ${ }^{22,23,35}$ a transmitted/ founder virus 1086.C gp120 and gp140; ${ }^{38} \mathrm{CH} 505$ gp120; ${ }^{39}$ a consensus clade C gp140 Env with deletions in the gp41 cleavage site, fusion domain and immunodominant region (CFI); Con-S gp140 CFI; gp41 sp400 peptide (RVLAVERYLRDQQLLGIWGCSGKLICTTAVPWN ASWSNKSLNKI); gp41 sp62 peptide (GGG-QQEKNEQELLELDK WASLWN); gp41 membrane proximal external region ${ }^{21}$ (CPC Scientific Inc., San Jose, CA); resurfaced stabilized core- $3^{5}$ produced by Drs Hua-Xin Liao and Barton Haynes; and HIV-1 MN recombinant gp41 (Immunodiagnostics, Woburn, MA).

HIV-1 Env-reactive antibodies were defined as having IgG quantitation $>0$ in small-scale transfection and an Env protein binding optical density of $\geq 0.13$ by ELISA, with background optical density $<0.06 .^{22,23,35}$ Percent reactivity for the blood and colostrum antibody repertoire of each individual was calculated as percent antibodies reactive by ELISA divided by the total number of antibodies produced from the compartment. Env specificities were classified as follows: gp41 (reactive against gp41, sp62, sp400, and/or membrane proximal external region; or gp41 and any gp140), gp120 (reactive against any gp120 protein, gp120 and any gp140, and/or resurfaced stabilized core-3), gp140 (reactive only against any gp140 protein), or multi-Env (reactive against both gp41 and gp120 proteins).

Breast milk supernatant HIV-1 neutralization and Env-specific IgG binding. Heterologous virus neutralization was performed on breast milk samples collected 4-6 weeks postpartum from all untreated subjects except $\mathrm{CH} 3601$, whose 4- to 6-week sample was not collected. The assays were performed in TZM-bl reporter cells with HIV-1 MW965.26, a clade C tier 1 virus, by methods described previously and are reported as $50 \%$ inhibitory dilution. ${ }^{13,40}$

HIV-1 Env-specific IgG levels were quantified from breast milk samples collected within 1 week of delivery for subject CH3601 and 4-6 weeks postpartum for all remaining untreated subjects using a customized HIV-1 binding antibody multiplex assay as described previously. ${ }^{13,22}$ HIV-1 Env-specific IgG responses were measured against a recombinant HIV-1 gp41 Env (Immunodiagnostics), multiclade consensus gp120 Env (Con-6 gp120), ${ }^{41}$ and Con-S gp140 CFI (from HX Liao) at a dilution of 1:500 (gp41 and gp140) or 1:100 (gp120). 2F5 was used as a positive control for gp41 and 2G12 as a positive control for gp120 and gp140. The coefficient of variation between duplicates was $\leq 20 \%$ and a minimum of 100 beads was counted per sample. Milk Env-specific IgG responses are reported as mean fluorescent intensity after blank beads subtraction.

Clonal B-Cell Lineage Analysis. Clonal relatedness among antibodies was assessed in two stages. First, candidate relatives were determined by shared $\mathrm{V}$ and $\mathrm{J}$ gene usage, and by a statistical test testing the hypothesis of a shared single CDR3 sequence, conditional on the mutation frequencies observed in the region encoded by the $\mathrm{V}$ gene. Second, confirmation was obtained through manual inspection for evidence suggesting independent rearrangements. The sequences were 
aligned using the BioEdit software (Ibis Biosciences, Carlsbad, CA), and the inferred unmutated common ancestor and intermediate heavy chain $\mathrm{V}(\mathrm{D}) \mathrm{J}$ sequences were determined by the Cloanalyst software (http://www.bu.edu/computationalimmunology/research/software/) and analyzed as described previously. ${ }^{36,39,42}$ Clonal lineage trees were created with the FigTree software (http://tree.bio.ed.ac.uk/software/ figtree/).

Statistics. Comparisons of B-cell phenotypes in colostrum and blood were performed using the non-parametric Wilcoxon matched-pairs signed rank test. Correlations between subjects' plasma or milk viral load, milk tier 1 neutralization, milk HIV-1 Env-specific IgG binding, and the proportion of HIV-1 Env-specific colostrum B cells were performed using Spearman's rank correlation. The threshold of inclusion of colostrum Env-specific B-cell frequency in these correlations was a total live B-cell population of greater than 100 cells. Rates of variable gene usage $\left(\mathrm{V}_{\mathrm{H}} 1^{\sim} 69\right.$ vs. other genes) and antigen specificity (gp41, gp120, or multi-Env vs. other Env) were compared between colostrum and blood antibodies using $2 \times 2$ contingency tables with Fisher's exact test. Somatic hypermutation frequencies and CDR3 lengths were compared between Env-reactive and non-reactive IgG antibodies in blood and colostrum using linear mixed models. Antibodies with identical heavy chains (duplicates) and heavy chains unable to be analyzed into constituent gene segments by Somatic Diversification Analysis (indeterminate sequences) were excluded from all statistical analyses. A $P$-value of $<0.05$ (two-tailed) was considered as significant for all analyses.

SUPPLEMENTARY MATERIAL is linked to the online version of the paper at http://www.nature.com/mi

\section{ACKNOWLEDGMENTS}

We acknowledge the following individuals for their contributions and support: Josh Amos, Sheri Chen, Annie Hogan, James Holland, Jennifer Kirchherr, Krissey Lloyd, Robert Parks, Christina Stolarchuk, Ashley Trama, Wilton Williams, and Yi Yang. Research reported in this publication was supported by the National Institute of Allergy and Infectious Diseases of the National Institutes of Health R01 Al106380 (Permar), the Howard Hughes Medical Institute (Sacha), the Center for HIV/AIDS Vaccine Immunology and Immunogen Discovery, grant number UM1-Al100645-01, and the Duke University Center for AIDS Research (CFAR), an NIH funded program (5P30 Al064518). The content is solely the responsibility of the authors and does not necessarily represent the official views of the National Institutes of Health.

\section{DISCLOSURE}

The authors declared no conflict of interest.

(c) 2015 Society for Mucosal Immunology

\section{REFERENCES}

1. UNAIDS. Fact Sheet: New HIV Infections [cited]. Available from http:// www.unaids.org/en/resources/campaigns/globalreport2013/factsheet/.

2. Rerks-Ngarm, S. et al. Vaccination with ALVAC and AIDSVAX to prevent HIV-1 infection in Thailand. N Engl J Med 361, 2209-2220 (2009).

3. Scheid, J.F. et al. Broad diversity of neutralizing antibodies isolated from memory B cells in HIV-infected individuals. Nature 458, 636-640 (2009).

4. Walker, L.M. et al. Broad and potent neutralizing antibodies from an African donor reveal a new HIV-1 vaccine target. Science 326, 285-289 (2009).

5. Wu, X. et al. Rational design of envelope identifies broadly neutralizing human monoclonal antibodies to HIV-1. Science 329, 856-861 (2010).

6. Morris, L. et al. Isolation of a human anti-HIV gp41 membrane proximal region neutralizing antibody by antigen-specific single B cell sorting. PLOS One 6, e23532 (2011).

7. Roux, M.E., McWilliams, M., Phillips-Quagliata, J.M., Weisz-Carrington, P. \& Lamm, M.E. Origin of IgA-secreting plasma cells in the mammary gland. J Exp Med 146, 1311-1322 (1977).
8. Kourtis, A.P., Butera, S., Ibegbu, C., Belec, L. \& Duerr, A. Breast milk and HIV-1: vector of transmission or vehicle of protection? Lancet Infect Dis $\mathbf{3}$, 786-793 (2003).

9. Perre PVd, Simonon A et al. Infective and anti-infective properties of breastmilk from HIV-1-infected women. Lancet 341, 914-918 (1993).

10. Rousseau, C.M. et al. Longitudinal analysis of human immunodeficiency virus type 1 RNA in breast milk and of its relationship to infant infection and maternal disease. J Infect Dis 187, 741-747 (2003).

11. Brandtzaeg, P. The secretory immune system of lactating human mammary glands compared with other exocrine organs. Ann. NY Acad. Sci. 409, 353-382 (1979).

12. Tuaillon, E. et al. Human milk-derived B cells: a highly activated switched memory cell population primed to secrete antibodies. J. Immunol. 182, 7155-7162 (2009).

13. Fouda, G.G. et al. HIV-specific functional antibody responses in breast milk mirror those in plasma and are primarily mediated by $\lg G$ antibodies. J. Virol. 85, 9555-9567 (2011).

14. Mabuka, J., Nduati, R., Odem-Davis, K., Peterson, D. \& Overbaugh, J. HIV-specific antibodies capable of ADCC are common in breastmilk and are associated with reduced risk of transmission in women with high viral loads. PLoS Pathog. 8, e1002739 (2012).

15. Becquart, P., Hocini, H., Garin, B., Sépou, A., Kazatchkine, M.D. \& Bélec, L. Compartmentalization of the IgG immune response to HIV-1 in breast milk. AIDS 13, 1323-1331 (1999).

16. Friedman, J. et al. Isolation of HIV-1-neutralizing mucosal monoclonal antibodies from human colostrum. PLOS ONE 7, e37648 (2012).

17. Kaminski, D.A., Wei, C., Qian, Y., Rosenberg, A.F. \& Sanz, I. Advances in human B cell phenotypic profiling. Front. Immunol. 3, 302 (2012).

18. Sanz, I., Wei, C., Lee, F.E. \& Anolik, J. Phenotypic and functional heterogeneity of human memory B cells. Semin. Immunol. 20, 67-82 (2008).

19. Kwong, P.D. \& Mascola, J.R. Human antibodies that neutralize HIV-1: identification, structures, and B cell ontogenies. Immunity 37, 412-425 (2012).

20. Haynes, B.F., Moody, M.A., Liao, H.X., Verkoczy, L. \& Tomaras, G.D. B cell responses to HIV-1 infection and vaccination: pathways to preventing infection. Trends Mol. Med. 17, 108-116 (2011).

21. Muster, T. et al. A conserved neutralizing epitope on gp41 of human immunodeficiency virus type 1. J. Virol. 67, 6642-6647 (1993).

22. Tomaras, G.D. et al. Initial B-cell responses to transmitted human immunodeficiency virus type 1: virion-binding immunoglobulin $\mathrm{M}$ (IgM) and IgG antibodies followed by plasma anti-gp41 antibodies with ineffective control of initial viremia. J. Virol. 82, 12449-12463 (2008).

23. Liao, H.X. et al. Initial antibodies binding to HIV-1 gp41 in acutely infected subjects are polyreactive and highly mutated. J. Exp. Med. 208, 2237-2249 (2011).

24. Yates, N.L. et al. HIV-1 gp41 envelope IgA is frequently elicited after transmission but has an initial short response half-life. Mucosal Immunol. 6 , 692-703 (2013).

25. Diaz-Jouanen, E. \& Williams, R.C. T and B lymphocytes in human colostrum. Clin. Immunol. Immunopathol. 3, 248-255 (1979).

26. Butler, J. \& Kehrli, M. Immunoglobulins and immunocytes in the mammary gland and its secretions. In: (Mestecky, J.F. \& Beinenstock, J. \& Lamm, M. \& Mayer, L. \& McGhee, J.R. \& Strover, W., (eds) Mucosal Immunology, 3rd edn (Academic Press, San Diego, 2004).

27. Permar, S.R. et al. Limited contribution of mucosal IgA to Simian immunodeficiency virus (SIV)-specific neutralizing antibody response and virus envelope evolution in breast milk of SIV-infected, lactating rhesus monkeys. J. Virol. 84, 8209-8218 (2010).

28. Belec, L. \& Kourtis, A.P. B lymphocyte-derived humoral immune defenses in breast milk transmission of the HIV-1. Adv. Exp. Med. Biol. 743, 139-160 (2012).

29. Salazar-Gonzalez, J.F. et al. Origin and evolution of HIV-1 in breast milk determined by single-genome amplification and sequencing. J. Virol. 85, 2751-2763 (2011).

30. Huang, C.C. et al. Structural basis of tyrosine sulfation and VH-gene usage in antibodies that recognize the HIV type 1 coreceptor-binding site on gp120. PNAS 101, 2706-2711 (2004).

31. Trkola, A. et al. Human monoclonal antibody $2 \mathrm{G} 12$ defines a distinctive neutralization epitope on the gp120 glycoprotein of human immunodeficiency virus type 1. J. Virol. 70, 1100-1108 (1996). 


\section{ARTICLES}

32. Janeway, C. The mucosal immune system. In Janeway's Immunobiology (Murphy, K. \& Travers, P. \& Walport, M., eds) 459-495, 7th edn (Garland Science, New York, NY, 2008).

33. Fouda, G.G. et al. Postnatally-transmitted HIV-1 Envelope variants have similar neutralization-sensitivity and function to that of nontransmitted breast milk variants. Retrovirology 10, 3 (2013).

34. Permar, S.R. et al. Potent SIV-specific cellular immune responses in the breast milk of SIV-infected, lactating rhesus monkeys. J. Immunol. 181, 3643-3650 (2008).

35. Liao, H.X. et al. High-throughput isolation of immunoglobulin genes from single human B cells and expression as monoclonal antibodies. J. Virol. Methods 158, 171-179 (2009).

36. Bonsignori, M. et al. Analysis of a clonal lineage of HIV-1 envelope V2N3 conformational epitope-specific broadly neutralizing antibodies and their inferred unmutated common ancestors. J. Virol. 85, 9998-10009 (2011).
37. Volpe, J.M., Cowell, L.G. \& Kepler, T.B. SoDA: implementation of a 3D alignment algorithm for inference of antigen receptor recombinations. Bioinformatics 22, 438-444 (2006).

38. Go, E.P., Liao, H.X., Alam, S.M., Hua, D., Haynes, B.F. \& Desaire, H. Characterization of host-cell line specific glycosylation profiles of early transmitted/founder HIV-1 gp120 envelope proteins. J. Proteome Res. 12, 1223-1234 (2013).

39. Liao, H.X. et al. Co-evolution of a broadly neutralizing HIV-1 antibody and founder virus. Nature 496, 469-476 (2013).

40. Montefirori, D.C. Evaluating neutralizing antibodies against HIV, SIV, and SHIV in luciferase reporter gene assays. Curr. Protoc. Immunol. Chapter 12, Unit 12.11 (2005).

41. Gao, F. et al. Antigenicity and immunogenicity of a synthetic human immunodeficiency virus type 1 group m consensus envelope glycoprotein. J. Virol. 79, 1154-1163 (2005).

42. Kepler, T.B. Reconstructing a B-cell clonal lineage: statistical inference of unobserved ancestors. F1000Res 2, 103 (2013). 